\title{
Automated bar detection in local disk galaxies from the SDSS The colors of bars ${ }^{\star}$
}

\author{
G. Consolandi

\begin{abstract}
Dipartimento di Fisica G. Occhialini, Università di Milano-Bicocca, Piazza della Scienza 3, 20126 Milano, Italy
\end{abstract} \\ e-mail: guido.consolandi@mib.infn.it
}

Received 14 June 2016 / Accepted 14 July 2016

\begin{abstract}
This paper describes an automatic isophotal fitting procedure that succeeds, without the support of any visual inspection of either the images or the ellipticity/position-angle radial profiles, to extract a fairly pure sample of barred late-type galaxies (LTGs) among thousands of optical images from the Sloan Digital Sky Survey (SDSS). The procedure relies on previous methods to robustly extract the photometrical properties of a large sample of local SDSS galaxies and is tailored to extract bars on the basis of their well-known peculiarities in their position angle and ellipticity profiles. This procedure was run on a sample of 5853 galaxies in the Coma and Local superclusters. The procedure extracted a color, an ellipticity and a position angle radial profile of the ellipses fitted to the isophotes for each galaxy. Examining the profiles of 922 face-on LTGs $(B / A>0.7)$ automatically, the procedure found that $\sim 36 \%$ are barred. The local bar fraction strongly increases with stellar mass. The sample of barred galaxies is used to construct a set of template radial color profiles to test the impact of the barred galaxy population on the average color profiles as previously shown in the literature and to test the bar-quenching scenario. The radial color profile of barred galaxy shows that bars are on average redder than their surrounding disk producing an outside-in gradient toward red in correspondence with their corotation radius. The distribution of the extension of the deprojected length of the bar suggests that bars have strong impact on the gradients of averaged color profiles. The dependence of the profiles on the mass is consistent with the bar-quenching scenario, i.e. more massive barred galaxies have redder colors (hence older stellar population and suppressed star formation) inside their corotation radius with respect to their lower mass counterparts.
\end{abstract}

Key words. galaxies: photometry - galaxies: evolution - galaxies: structure - techniques: photometric

\section{Introduction}

Hydrodynamical simulations have made clear that bars have a major impact on the secular evolution of galaxies (Athanassoula 2002; Sellwood 2014). It is well known that barred potentials exert nonaxisymmetric forces onto the gaseous component of the galaxy. The gas within the corotational radius is rapidly funneled to the center of the galaxy, within the Inner Lindblad Resonance (see Kormendy \& Kennicutt 2004; Kormendy 2013; Sellwood 2014; Fanali et al. 2015), while the gas outside is confined to the outer disk (Sanders \& Huntley 1976; Shlosman et al. 1989; Athanassoula 1992; Berentzen et al. 1998; Regan \& Teuben 2004; Kim et al. 2012; Cole et al. 2014). While it is not clear whether this phenomenon can trigger AGN activity or not (Emsellem et al. 2015), it is out of the question that the high density reached by the gas dragged into the center of the galaxy triggers a burst of star formation that rapidly depletes it, making the region inside the corotational radius gas-poor (Krumholz \& McKee 2005; Krumholz et al. 2009; Daddi et al. 2010; Genzel et al. 2010). From an observational point of view, starting from the seminal work of E. Hubble who dedicated half of the world-famous tuning fork to barred disk galaxies (Hubble 1936), bars have increasingly captured the interest in the understanding of galaxy secular evolution. As a

\footnotetext{
* Tables of the barred and non-barred galaxies are only available at the CDS via anonymous ftp to

cdsarc.u-strasbg. fr (130.79.128.5) or via

http://cdsarc.u-strasbg.fr/viz-bin/qcat?J/A+A/595/A67
}

matter of fact, throughout the years observations have enlighten the physical effects of bars on galaxies (Sakamoto et al. 1999; Kormendy \& Kennicutt 2004; Jogee et al. 2005; Sheth et al. 2005; Kormendy 2013) and, above all, the extremely high frequency of barred galaxies among spirals. Indeed, among local bright disk galaxies, 60\% (Knapen 1999; Eskridge et al. 2000; Menéndez-Delmestre et al. 2007; Marinova \& Jogee 2007) are barred if observed in the near-infrared and about $\sim 40 \%$ are barred if observed in the optical bands (Eskridge et al. 2000; Marinova \& Jogee 2007), which hints at bars as fundamental drivers of the evolution of late-type galaxies (LTGs).

If and how the bar fraction evolves across the cosmic time (Jogee et al. 2004; Sheth et al. 2008), along with the exact determination of the dependence of the bar frequency on stellar mass (especially at the faint end of the mass function) and galaxy environment, are still under debate (Thompson 1981; Marinova et al. 2012; Skibba et al. 2012; Lansbury et al. 2014; Alonso et al. 2014). For example, Masters et al. (2012), Skibba et al. (2012), Méndez-Abreu et al. (2012), and Gavazzi et al. (2015) all consistently report a bar fraction that increases with increasing mass. Nevertheless, Barazza et al. (2008) recover a strong bar fraction that increases with decreasing mass while Nair \& Abraham (2010b) find a strong bar fraction that decreases from $\sim 10^{9} M_{\odot}$ to $\sim 10^{10} M_{\odot}$ and increases again from $\sim 10^{10} M_{\odot}$ to $\sim 10^{11} M_{\odot}$.

The work by Cheung et al. (2013) and Gavazzi et al. (2015) underline the crucial importance of determining the exact dependence of the fraction of strongly barred galaxies on total stellar mass. Namely, an increasing bar fraction with increasing 
mass would explain the central quenching of the star formation (SF) in high mass main-sequence galaxies, which bends the local star formation rate (SFR) versus stellar mass relation at $\sim 10^{9.5} M_{\odot}$. Moreover, Sánchez-Janssen et al. (2010) report that below $\sim 10^{9.5} M_{\odot}$ disks are systematically thicker, making it difficult to develop bars.

Determining robustly the real bar fraction below $10^{9} M_{\odot}$ demands statistics as well as sensitivity and resolution. Both of these are lacking in high redshift determinations and the best environment to determine the optical bar fraction in such a wide range of mass is therefore the local Universe, taking advantage of the publicly available data of the Sloan Digital Sky Survey (SDSS, York et al. 2000). The SDSS fully covered the area of two nearby structures such as the Virgo and the Coma superclusters, which provide the opportunity to study thousands of galaxies down to a limiting mass as low as $10^{7} M_{\odot}$ with a physical resolution of $\sim 600 \mathrm{pc}$ at the distance of Coma. However, the task is non-trivial, as stellar bars among the population of dwarf LTGs are often low surface brightness features that are easily confused with or hidden by poorly resolved patches of SF. Purely visual inspection drives the classification of barred galaxies of most of the catalogs of galaxies such as the cases of the RC3 (de Vaucouleurs et al. 1991), VCC (Binggeli et al. 1985) and Nair \& Abraham (2010a) classifications. Nevertheless, because of the subjective nature of visual inspection classification, it is habitual to average the votes of as many classifiers as possible. A clear example is given by the recent works by Masters et al. (2011), Masters et al. (2012), Skibba et al. (2012), and Melvin et al. (2014) which are based on the classification of the Galaxy Zoo (GZ) project ${ }^{1}$ (see Lintott et al. 2008; Masters et al. 2011). This classification is made on the basis of thousands of votes assigned by citizens that are asked to visually inspect SDSS galaxies and to answer questions about the morphology of the object.

On the other hand, a different approach is adopted for example by Wozniak et al. (1995), Jogee et al. (2004), Barazza et al. (2008), Aguerri et al. (2009), and Méndez-Abreu et al. (2012), who support the visual inspection by performing isophotal fitting analysis and looking at some precise features in the ellipticity and position angle (PA) radial profiles.

This paper tests a different method of producing an objective classification that does not rely on visual inspection of either the images or the ellipticity/PA radial profiles of galaxies and extracts a fairly pure sample of barred LTGs. We ran an automatic procedure, based on the sample and on the methods explained in Consolandi et al. (2016) for the isophotal fitting (explained in Sect. 3), on $~ 6000$ SDSS galaxies in the Local and Coma superclusters. Section 3.2 is presents the automatic bar-extraction criteria based on the ellipticity and PA profiles extracted. Limitations, pureness, and completeness of the automatic classification are discussed in Sect. 4 by comparing the final selection to other classifications found in the literature. In Sect. 5 the resulting bar fraction versus mass relation is compared to other published results. Finally, the results are discussed in Sect. 6 and the bar-quenching scenario described by Gavazzi et al. (2015) is tested by creating a set of templates of color profiles of barred galaxies in different bins of mass. These are also compared to the template color profiles produced in Consolandi et al. (2016, from now on C16).

\footnotetext{
wWw.galaxyzoo.org
}

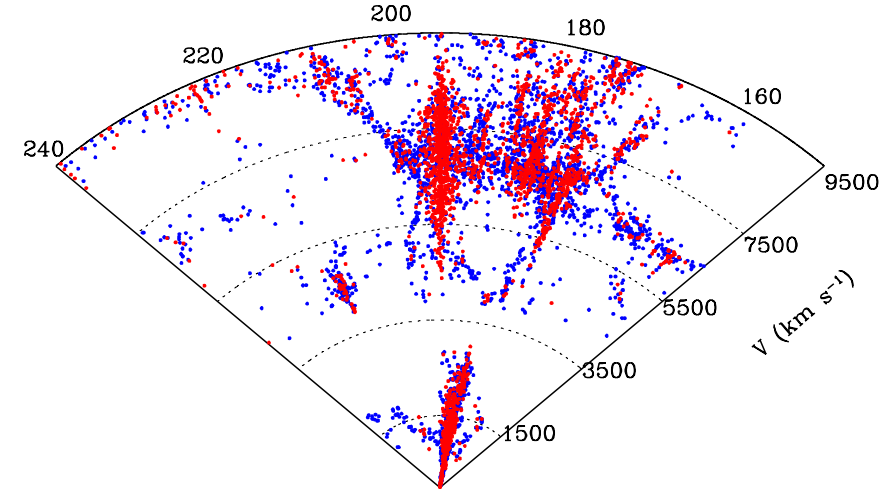

Fig. 1. Wedge diagram of galaxies belonging to the sample studied in this work: the Coma Supercluster $\left(c z>4000 \mathrm{~km} \mathrm{~s}^{-1}\right)$ and the Local Supercluster $\left(c z<3000 \mathrm{~km} \mathrm{~s}^{-1}\right)$. Blue dots represent late-type galaxies while red dots stand for early-type galaxies.

\section{The sample}

The sample analyzed in this work is shown in Fig. 1 and coincides with the sample presented by $\mathrm{C} 16$. Our sample comprises 6136 nearby galaxies in the spring sky selected from the SDSS and further split in two subsamples: the Local Supercluster $\left(11^{\mathrm{h}}<\mathrm{RA}<16^{\mathrm{h}} ; 0^{\mathrm{o}}<\right.$ Dec $\left.<18^{\mathrm{o}} ; \mathrm{cz}<3000 \mathrm{~km} \mathrm{~s}^{-1}\right)$ which contains 1112 galaxies and includes the Virgo cluster; and the Coma Supercluster $\left(10^{\mathrm{h}}<\mathrm{RA}<16^{\mathrm{h}} ; 18^{\mathrm{o}}<\right.$ Dec $<32^{\mathrm{o}}$; $4000<c z<9500 \mathrm{~km} \mathrm{~s}^{-1}$ ), which contains 5024 galaxies and includes the Coma cluster. Since galaxies at the distance of Virgo have apparent size often exceeding 5 arcmin, they are strongly affected by the shredding problem (Blanton et al. 2005), and therefore, our catalog cannot solely rely on the SDSS spectroscopic database. Briefly, the Local Supercluster sample is selected following the prescriptions of Gavazzi et al. (2012): in the area occupied by the Virgo cluster, the selection is based on the VCC catalog (limited however to $c z<3000 \mathrm{~km} \mathrm{~s}^{-1}$ ) down to its magnitude completeness limit of 18 mag (Binggeli et al. 1985). The object selection, furthermore, is limited to objects with surface brightness above the $1 \sigma$ of the mean sky surface brightness in the $i$ band of the SDSS data (C16). Outside the Virgo cluster the SDSS selection is complemented with objects taken from NED and ALFALFA (Haynes et al. 2011). At the distance of the Coma Supercluster the shredding problem is instead less severe and therefore we followed the selection criteria of Gavazzi et al. (2010, 2013): galaxies are selected from the SDSS spectroscopic database DR7 (Abazajian et al. 2009) with $r<17.77$ mag and to fill the residual incompleteness of the SDSS catalog for extended galaxies and due to fiber conflict, 133 galaxies from the Catalog of Galaxies and Clusters of Galaxies (CGCG; Zwicky et al. 1968 ) with known redshifts from NED and 28 from ALFALFA are added, reaching a total of 5024 galaxies.

For these galaxies, C16 downloaded the SDSS images (using the on-line Mosaic service, Berriman et al. 2004) only in the $g$ and $i$ band for mainly two reason. First, their higher signal-tonoise ratio $(\mathrm{S} / \mathrm{N})$ compared to the $u$ and $z$ filters and, second, the $r$ filter have a central wavelength that is closer to the $g$ filter central wavelength with respect to the $i$ filter, making the $g-i$ color more sensitive to stellar population gradients. The download process worked in both the $i$ and the $g$ band for 5753 (94\%) targets out of which, 221 galaxies were discarded a posteriori because they lie too close to bright stars or they have a surface brightness that is too low.

The remaining analyzed sample constitutes 5532 galaxies that can be considered representative of the nearby Universe. The 


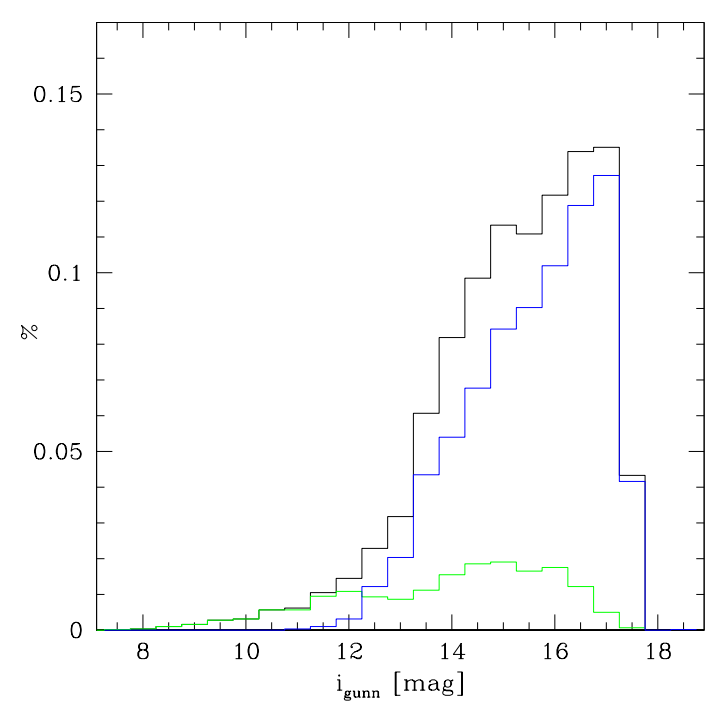

Fig. 2. Distribution of the $i$-band magnitudes of the whole sample; separate distribution for the Local (green line) and the Coma (blue line) supercluster samples.

distribution of the $i$-band magnitudes of this sample is shown in Fig. 2. Galaxy masses are computed assuming a Chabrier IMF, following Zibetti et al. (2009) from the $i$-band luminosities of the galaxies and their $(g-i)$ color published in C16.

\section{Automatic tilted profiles for nonaxisymmetric structure detection}

C16 presented a semi-automated IDL procedure that performs photometry and extracts surface brightness and color profiles over multi-band SDSS images of local galaxies. Briefly, stamp images ( $g$ and $i$ band) centered on target galaxies are downloaded from the on-line Mosaic service (Berriman et al. 2004). The procedure automatically performs sky subtraction in each filter and creates an averaged white image with an improved signal-to-noise ratio. This image is processed by Source Extractor (Bertin \& Arnouts 1996), which detects stars and galaxies producing a map of the fore- and background objects that is exploited to create an accurate mask of the field of view. Simultaneously, the external geometry of the galaxy is extracted by Source Extractor and exploited by our routine to create (in the white frame) a set of concentric ellipses fitting the target and enabling the evaluation of the surface brightness profile in the $g$ and $i$ images and the $g-i$ color profile. The latter are therefore extracted using ellipses with fixed ellipticity and position angle and do not retain any imprint of a possible bar component, such as the twist of the isophotes or a peak in the ellipticity profile.

In this work, I describe the implementation of an accessory IDL-based routine of the $\mathrm{C} 16$ procedure that is able to quickly perform ellipse fitting (Jogee et al. 2004; Marinova \& Jogee 2007; Aguerri et al. 2009) and automatically extract the barred galaxies among the sample. This tool makes use of two fundamental outputs of the $\mathrm{C} 16$ procedure: the mask of fore- and background objects, the white light image and the white light surface brightness profile. Eventually, for each galaxy, the new routine returns the ellipticity and PA of the fitted ellipses as a function of the semi-major axis and the surface brightness and color profiles derived from the same ellipses.

\subsection{Ellipse fitting}

In order to correctly evaluate color profiles, surface brightness profiles must be extracted over consistent apertures in the $g$ and $i$ bands. The ellipse fitting procedure is performed on the white image that represents a high $\mathrm{S} / \mathrm{N}$ reference frame common to all filters. For each galaxy, the procedure fits ellipses to the contours of the white light frame, which are built based on the cgContour routine of the IDL Coyote Library. The routine is set to extract 100 logarithmic levels ranging from $3 \times \sigma_{\text {sky }}$ to the maximum of the white surface brightness (non-tilted) profile, which was previously evaluated by the procedure described in C16. The cgContour routine returns the two-dimensional distribution of pixels of each contour level in the white frame. Starting from outside the galaxy and going toward the center, the procedure automatically fits each contour with the parametric formula of an ellipse rotated of a position angle (PA; each ellipse is fitted owing to the mpfitellipse procedure that is based on the mpfit routine, a non-linear least squares fitting program described in Markwardt 2009). For each contour level I setup the initial guesses for the ellipse-fitting assuming the center $X_{\mathrm{c}}, Y_{\mathrm{c}}$ of the ellipse as the average $X, Y$ pixel coordinates composing the contour level distribution. Further on the initial guesses for the semi-major and semi-minor axis are chosen as half the distance between the first (/half-path) pixel coordinates and the one at half-path (/three quarters), while the initial guess for the PA is taken as 0. Each fitted ellipse must not overlap the previously fitted ellipse. Once all contours are fitted, the routine checks and discards ellipses that are not centered. To this aim, the coordinates of the center previously evaluated by Source Extractor are not considered and are instead recomputed as the mode of the coordinates of the center of all fitted ellipses ${ }^{2}$.

The procedure discards ellipses whose center are at a distance greater than $\sim 15$ arcsec from the galaxy center for Virgo galaxies and $\sim 5$ arcsec for Coma. Moreover, in some cases, especially among irregular galaxies, the most internal ellipses fits contours that follow patches of star formation that are not centered on the galaxy but are still within the tolerance distance adopted. To fix this, ellipses that have central coordinates at a distance to the galaxy center greater than their semi-major axis (sma) are not considered.

Finally, the surface brightness of each ellipse in the $g$ and $i$ band images are computed measuring the average surface brightness along the path of each fitted contour, and $(g-i)$ color profiles are consequently obtained making the difference between the $g$ and $i$ surface brightness profiles. Examples of the ellipses fitted and of the profiles extracted by the procedure are shown in Fig. 3 for four different galaxies (three barred and one unbarred).

\subsection{Bar selection}

As it is described by Wozniak et al. (1995), Jogee et al. (2004), Marinova \& Jogee (2007), Barazza et al. (2008), and Aguerri et al. (2009), and as can be seen in Fig. 3, bars in faceon galaxies produce a peak in the ellipticity profile to which a plateau in the PA is associated, i.e., the PA is constant within $\pm 20^{\circ}$. The ellipse fitting method has been extensively tested by many authors to be efficient in extracting barred galaxies among wide samples (Jogee et al. 2004; Marinova \& Jogee 2007; Aguerri et al. 2009; Laurikainen et al. 2010). This fitting

2 This choice was made because the coordinates evaluated by Source Extractor are referred to the ellipse that fits the external (at the $1.5 \Sigma_{\text {sky }}$ isophote) geometry of the galaxy which does not always provide the correct barycenter of the internal isophotes. 

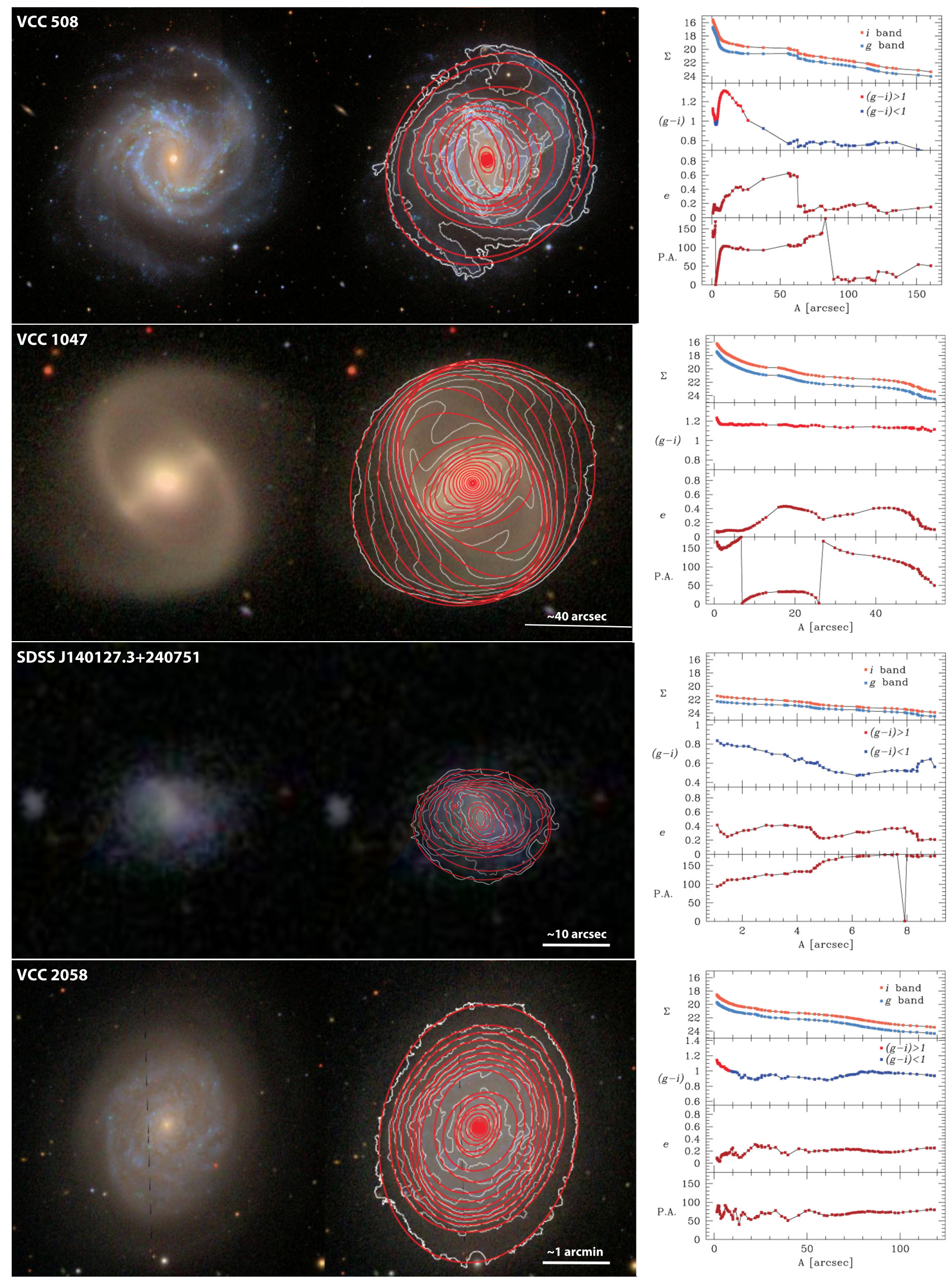

Fig. 3. Three barred galaxies and one unbarred VCC-508, VCC-1047, SDSS-J140127.3 + 240751, and VCC-2058, respectively. For each object, the surface brightness and color profile, as well as the ellipticity and PA of the fitted ellipses as a function of the semi-major axis, are plotted. 
has two free parameters, $\Delta e$ and $\Delta \mathrm{PA}$. The first parameter is the difference between the ellipticity of the bar and that of the disk, while the second parameter is the interval within which the PA varies along the bar.

The procedure analyzes the properties of the ellipticity and PA profiles of each galaxy and hunts bars automatically extracting those objects that exhibit $\Delta e>0.08$ and $\Delta \mathrm{PA}>20^{\circ}$. These threshold values were tested by Aguerri et al. (2009) who showed that these values minimize the fraction of spurious/bad detections and simultaneously maximize the bar identifications. In order to automatically select galaxies on these bases, the procedure must correctly identify the ellipticity peak and the related PA plateau associated with the bar. Obviously both variations of the ellipticity and PA are more evident and easier to detect in face-on galaxies, where the contrast between the geometry of the galaxy and a bar is maximum. Hence we limited the extraction to galaxies with $B / A>0.7$, where the axis ratio $B / A$ is evaluated by the procedure itself as the average ratio between the semi-minor (B) and semi-major (A) axes of the four most external ellipses fitted. The blind extraction happens in three distinct phases: in the first phase (i), the procedure finds each ellipticity peak in the ellipticity profile and, for each of them, looks for a related plateau in the PA profile; in a second instance (ii), the ellipticity peaks associated with a plateau are compared and the most promising peak is extracted; and finally (iii), the geometry of the isophote associated with the extracted peak is compared to the galaxy geometry and the final word on the possible bar presence is made. We outline the steps of the procedure below.

Step (i): in order to identify the plateau, for each peak the routine considers all fitted isophotes in the neighborhood of each considered peak with PA within $20^{\circ}$ from $\mathrm{PA}_{\text {peak }}$ an ellipticity within 0.1 from $e_{\text {peak }}$.

Step (ii): peaks found in (i) are all thought to represent a possible bar and are therefore placed into competition. Each peak receive a positive or negative vote according to their ellipticity, $\Delta e$, length of the plateau, and number of fitted ellipses. In other words, the procedure gives the best votes to the peaks with the greatest ellipticity that 1) maximizes the ratio between the length of the plateau and the sma at which the peak is found; 2) minimizes the variations in the PA profile; and 3 ) is found in the region $0.05<a_{\text {peak }} / a_{\text {gal }}<0.95$. Eventually only the best ranked peak is considered and if two (or more) peaks get the same vote, the preference is given to the most internal peak ${ }^{3}$.

Step (iii): once that a single-peak is extracted, its vote is further boosted according to its values of $\Delta e$ and $\Delta \mathrm{PA}$, i.e., a peak that has $\Delta e \sim 0.4$ and $\Delta \mathrm{PA} \lesssim 10$ receive a higher rank compared to a peak that has $0.1 \lesssim \Delta e \lesssim 0.2$ and $10 \lesssim \triangle \mathrm{PA} \lesssim 20$.

\section{Selected bars}

In order to account only for systems that can develop nonaxisymmetric structures in the barfraction estimate, a morphological cut is applied to exclude pressure supported systems. The morphological classification of all the galaxies of the sample is found in the online public database GOLDMine (Gavazzi et al. 2003, 2014). Nevertheless this classification is purely visual

\footnotetext{
3 As a matter of fact, spiral arms can produce a peak in the ellipticity profile and a PA plateau that mimes the behavior of a bar, but when the bar is present they have larger scale length.
}

Table 1. Frequencies of bad, uncertain, and confirmed detections after visual inspection of the 922 face-on LTGs.

\begin{tabular}{cccc}
\hline \hline & $N_{\text {gal }}$ & Bad classification & Uncertain \\
\hline vote $\leq 0$ & 557 & 22 & 10 \\
vote $>0$ & 365 & 30 & 25 \\
vote $=1$ & 32 & 10 & 4 \\
vote $=2$ & 24 & 3 & 4 \\
vote $=3$ & 66 & 5 & 4 \\
vote $=4$ & 16 & 2 & 2 \\
vote $\geq 2$ & 333 & 18 & 21 \\
vote $\geq 4$ & 227 & 10 & 11 \\
\hline
\end{tabular}

Notes. Bad detections are the missed bars, in the category with a vote lower than 0 , or, in the other groups, the unbarred galaxies considered as barred.

(Binggeli et al. 1985) and probably does not accurately separate slowly rotating systems (elliptical galaxies) and fast rotators (namely S0s), preventing a robust estimate of the bar fraction when including early-type disks. Therefore I give the results separately for the sample of late-type disks (from S0a to Sm and Irr) and the sample that includes S0s. The procedure performed the extraction over a subsample of 922 face-on $(B / A>0.7)$ LTGs (from S0a to Sm and Irr) and over 447 S0s and dS0s. The sample including early-type disks therefore accounts for 1365 members. In the late-type disks sample 365 galaxies received a positive vote (442 including early types) and, thus, have been highlighted to be possible bars. A visual inspection of all galaxies revealed that above vote $=4$ (227 galaxies) the sample of bars extracted can be considered more than $95 \%$ pure while below vote $=1$ (557 galaxies) only $\sim 20$ barred galaxies were erroneously classified as unbarred (3-4\%). The votes $=2,3,4$ categories (106) comprehend about $10 \%$ of bad detections and $~ 9 \%$ of ambiguous cases in which, even after visual inspection of both images and profiles, it is very hard to speak a final word on their real morphology. This is summarized in Table 1, which indicates that selecting galaxies with vote $\geq 2$ defines a satisfactory $\sim 90 \%$ (considering as intruders both bad and uncertain cases) pure sample of barred galaxies, which maximizes the overall bar fraction of our sample $(36 \% \pm 2 \%)$.

\subsection{Comparison with visual classifications}

To further test the efficiency of the extraction of barred objects, I compared this classification to that performed by Binggeli et al. (1985) for galaxies of the Virgo Cluster Catalog (VCC). Among the VCC, face-on LTGs with a $B / A>0.7$ and a $c z<3000 \mathrm{~km} \mathrm{~s}^{-1}$ are 56, out of which 20 are classified as barred. Among VCC bars, $80 \%$ have also been consistently classified as barred by my automatic method which, on the other hand, classifies 16 more galaxies (among which there is VCC 508, see Fig. 3a) as barred and misses 4 barred galaxies from the VCC, thereby implying an overall accordance between the present classification and Binggeli of $\$ 70 \%$. Nevertheless, among the objects classified as barred by the automatic pipeline and unbarred by the VCC, $50 \%$ are instead classified as barred in the RC3 (de Vaucouleurs et al. 1991), which indicates that the bar extraction efficiency of the pipeline is better than what we can deduce from the accordance with VCC. The remaining 50\% (9 galaxies) are either classified as peculiar objects or weakly barred; these are mostly low surface brightness systems with some degree of perturbation and 
asymmetry. Only 4 galaxies do not show any bar component and are classified as normal LTGs by both RC3 and VCC.

A more recent classification based on SDSS images was performed over a sample of about 500 galaxies in the Virgo cluster by Méndez-Abreu et al. (2012) and over a different sample of about 200 galaxies in the Coma cluster by Méndez-Abreu et al. (2010). I found a sample of 452 objects in common with the sample of this work and those of Méndez-Abreu et al. (2012). Performing our selection cut in their sample leads to a test sample of 29 galaxies out of which 14 are barred in the Méndez-Abreu et al. (2012) classification. Among these, 12 have been consistently voted as barred galaxies by my automatic procedure, while 2 galaxies where erroneously considered unbarred and another 2 are not classified as barred in the Méndez-Abreu et al. (2010, 2012) classifications. If we instead include the analysis of S0s, we find a test sample of 50 galaxies. In this case Méndez-Abreu et al. (2010) finds 23 barred objects while the automatic procedure extracts a total of 18 bars. Overall, 17 galaxies were classified as barred by both works, while 6 galaxies were missed by my automatic procedure. The overall agreement in the classification of the 50 objects selected is $\sim 82 \%$.

Finally we compare the classification with that extracted in the GZ (Lintott et al. 2008; Willett et al. 2013), selecting objects with pbar $>0.5$ and that have been voted by more than 28 citizens (Melvin et al. 2014). In total I found a GZ classification for 727 LTGs with $B A>0.7$ out of which 180 barred (25\%). On the contrary our procedure finds 285 barred galaxies $(\sim 39 \%)$ including $80 \%$ of the galaxies classified as barred by the zoo. The objects that are not found barred in the GZ project are mostly (62\%) galaxies with $M_{*}<10^{9.5} M_{\odot}$. In these cases, the procedure is sensitive to the nonaxisymmetric distribution of bright HII regions and clumps in these irregular galaxies, leading to wrong or ambiguous detections that overestimate the fraction in the low mass systems. On the other hand, among brighter galaxies, it was possible to find an independent classification of the RC3 and/or VCC catalogs, indicating $~ 30 \%$ of the objects voted unbarred in the GZ are in fact barred. This indicates the good performance of the procedure at higher luminosities. Moreover about $50 \%$ of the remaining galaxies have a pbar between 0.35 and 0.5 , indicating that they were nearly recognized as barred in the GZ. Therefore there is evidence that the present procedure overestimates the fraction at low mass but performs very well at higher masses, for an overall agreement with this classification of $\sim 78 \%$.

In spite of the aforementioned difficulties of this automatic method at low mass, the overall agreement among the classifications is satisfactory but with an high source of uncertainty coming from bars detected in low mass systems. These are often low surface brightness with poorly resolved structures that, even after visual inspection of their images and ellipticity profiles, it is very difficult to say if they harbor bars (see Fig. 3, the galaxy SDSSJ140127.3+240751).

A further demonstration comes from the comparison with the visual classification performed in Gavazzi et al. (2015) by all the authors. In total, I could compare the classification of 229 galaxies out of which 51 were classified as barred by the authors. In this work the procedure was able to spot $\sim 80 \%$ of the bars classified in Gavazzi et al. (2015) but, on the other hand, the procedure classifies 60 more bars. Of these, the $68 \%$ are once again low-mass $\left(M_{*}<10^{9.5} M_{\odot}\right)$ irregularly shaped systems. Of the remaining more massive galaxies that were not classified as bars by Gavazzi et al. (2015), 33\% are confirmed to host a bar by other independent classifications found in NED that

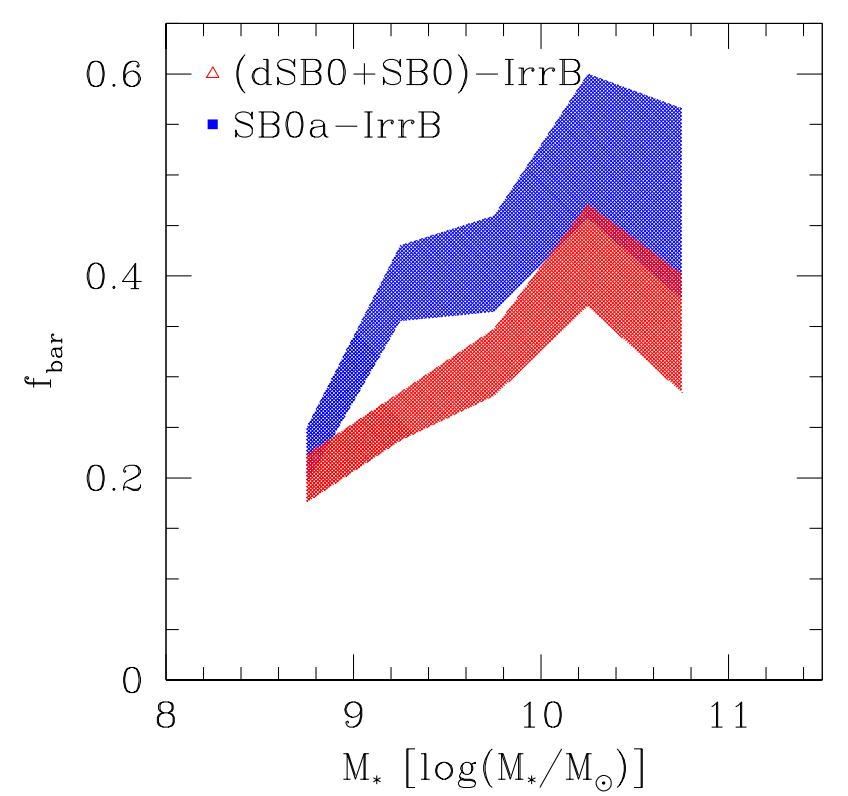

Fig. 4. Optical local bar fraction as a function of stellar mass given separately for the sample that includes galaxies from SOa to Irr (blue) and the sample that accounts for SB0s (red). The width of the shaded area gives the poisson uncertainty in each bin.

corroborate the result of this method. Once again, the procedure shows a good capability in spotting bars in high luminosity systems, while having more difficulty at low mass.

\section{The local bar fraction}

In the past section we described our selection and showed that the procedure selected a reliable sample of barred galaxies. C16 showed that spiral galaxies above some threshold mass are undoubtly redder then their lower mass counterparts and that this phenomenon is more evident in their central parts. I now test the consistency of the evaluated bar fraction with the bars extracted by Méndez-Abreu et al. (2012) and Gavazzi et al. (2015) and then check whether the presence of a bar can indeed produce the trend in color profiles with mass that C16 observed by building a template color profile of barred galaxies.

The overall local bar fraction evaluated in the present analysis is $36 \%$, while if we include S0s it becomes $28 \%$. The agreement with Jogee et al. (2004), Marinova \& Jogee (2007), Barazza et al. (2008), Nair \& Abraham (2010b), Oh et al. (2012), and Gavazzi et al. (2015) is satisfactory. Figure 4 shows the local bar fraction as a function of mass obtained separately for LTGs and LTGs plus S0s and dS0s. This has been evaluated in five bins of 0.5 dex from $10^{8.5} M_{\odot}$ to $10^{11} M_{\odot}$. The bar fraction increases evidently with increasing mass in both relations confirming many literature results (Méndez-Abreu et al. 2012; Masters et al. 2012; Skibba et al. 2012; Cheung et al. 2013; Gavazzi et al. 2015). Nevertheless there is a clear separation between the sample containing lenticular galaxies and the LTG-only sample. Compared to the bar fraction published by Gavazzi et al. (2015), the fraction at low mass is considerably higher. As discussed in the previous section, the automatic pipeline is very sensitive to irregular structures in low mass galaxies and may produce an higher fraction at low mass. In this sense, the low mass end of this bar fraction could be considered an upper limit. Nevertheless, the steep relation between the total stellar mass and the bar occupation fraction is preserved. 
G. Consolandi: Automated bar detection in local disk galaxies from the SDSS

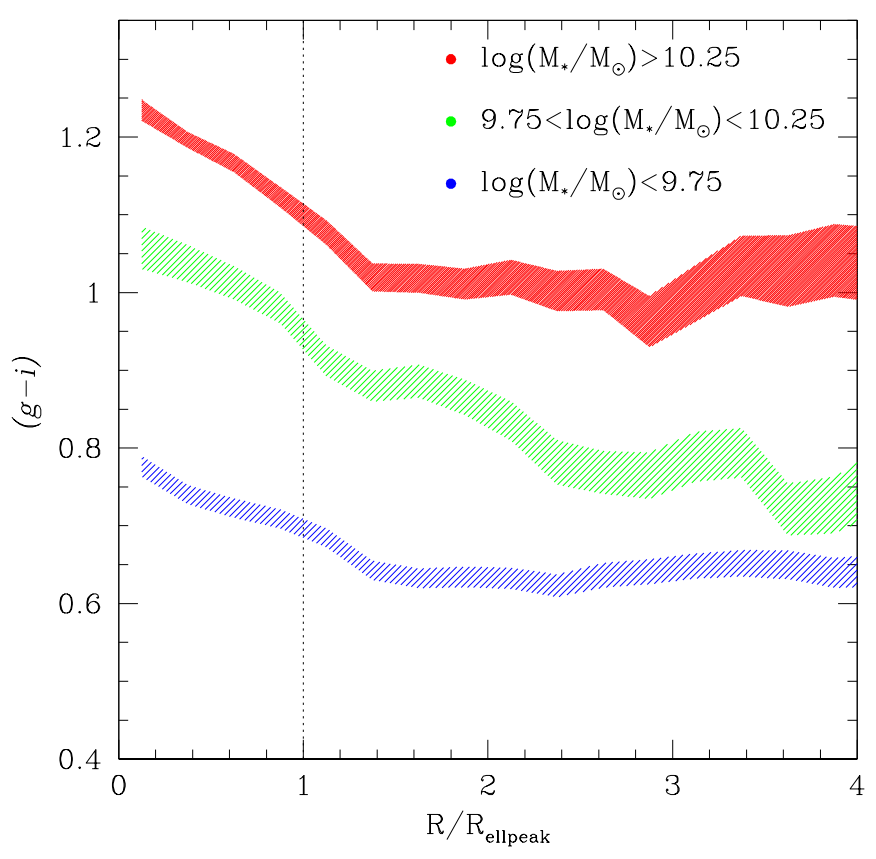

Fig. 5. Color profiles templates of barred galaxies automatically extracted in this work in three bins of increasing mass.

\section{The colors of bars: discussion and conclusions}

Maintaining the selection onto LTGs and excluding S0s, I constructed a template $(g-i)$ color profile in three different bins of mass: (i) below $M<10^{9.75} M_{\odot}$ (ii) $M>10^{9.75} M_{\odot}$ and $M<$ $10^{10.25} M_{\odot}$ (iii), and $M>10^{10.25} M_{\odot}$. The bins were selected to guarantee that more than 50 galaxies contribute to each template profile. Color profiles are good tracers of the specific SFR and works such as MacArthur et al. (2004), McDonald et al. (2011), and $\mathrm{C} 16$ highlighted the good correspondence between average age of the stellar population as a function of radius and color radial profiles. Moreover, using the technique of template profiles, C16 showed that massive spiral galaxies develop a red and dead component, the importance of which increases with mass. Using the same technique on the tilted color profiles of the subsample of barred galaxies of $\mathrm{C} 16$ extracted in this work, we test whether the central red and dead component is consistent with the presence of a bar-like structure in the center of galaxies. The template profiles for barred galaxies are shown in Fig. 5 with each radius normalized to the radius of the selected ellipticity peak ( $\left.R_{\text {ellpeak }}\right)$, which is a good proxy for the bar length and corotation radius (Laurikainen et al. 2010), with a radial step of $0.1 R / R_{\text {ellpeak }}$.

The template profiles evolve significantly from low to high mass. In the lowest mass bin, the color profile is blue over all radii, with possibly only a mild gradient toward red inside the bar radius. Things change clearly in the intermediate mass template profile: the outer disk $\left(R / R_{\text {peak }}>1\right)$ is blue and, in the outermost regions, it overlaps with the lowest mass bin profile, while inside the corotation radius $\left(R / R_{\text {peak }}<1\right)$ the color profile is red as an elliptical of the same mass. The bar has already reached the red sequence and, on the contrary, the disk is still on the blue cloud. In the highest mass bin, the red component once again has the typical color of the red sequence in the same range of mass. The disk is still bluer in the outer region but displays an average color of the disk that is redder than the respective average blue cloud values of the same mass. Moreover in Fig. 6 we show the template radial color profiles of barred galaxies for two samples above and below the threshold mass found by Gavazzi et al. (2015) to be the mass above which bars have

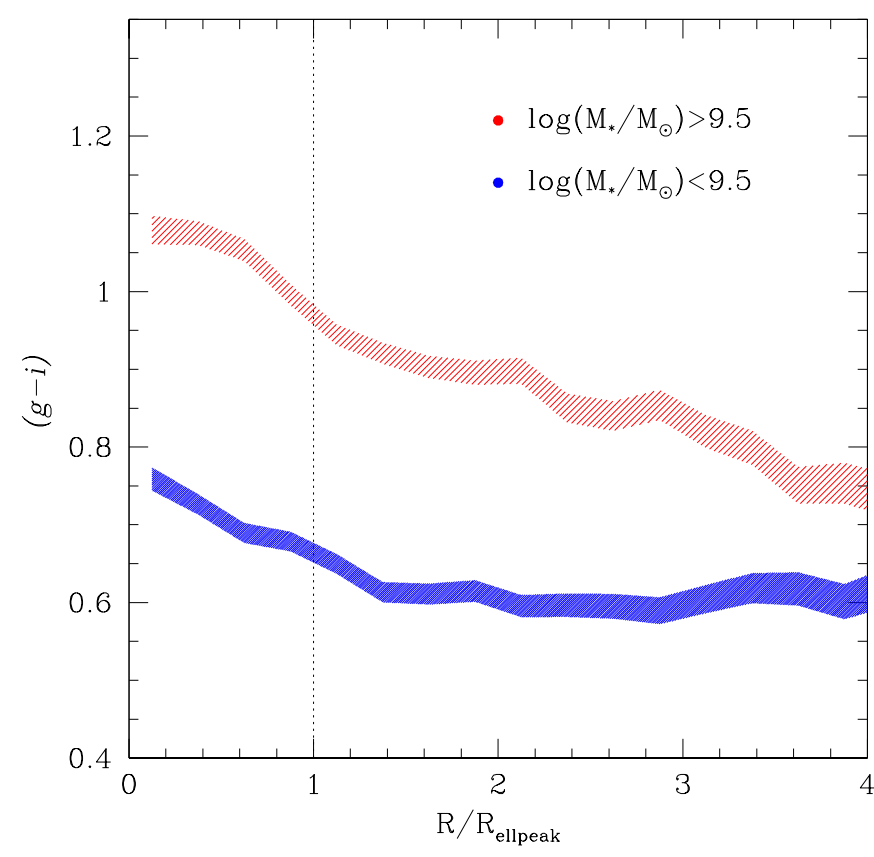

Fig. 6. Color profiles templates of barred galaxies automatically extracted in this work in two bins of increasing mass: above (red) and below (blue) $M_{\text {knee }}$, the threshold mass found by Gavazzi et al. (2015).

quenched the region inside the corotation radius and therefore red. In order to quantify the average extension of the region that is under the bar influence, we correct the radius of the ellipticity peak for projection effects using the measured PA with respect to its galaxy that is considered to have the PA of the last fitted isophote. The distribution of the ratio of the deprojected bar semi-major axis and the galaxy semi-major axis is plotted in Fig. 7. This distribution peaks at 0.3 consistently with others results, such as that published in Marinova \& Jogee (2007) and Barazza et al. (2008).

The bars that we extracted are primarily strong bars $(e>0.4$, Laurikainen et al. 2010) and weak bars represent only $9 \%$ of the bars extracted which is again consistent with the proportion observed in Marinova \& Jogee (2007).

Figure 5 allows us to deduce that bars are on average redder structures when they are compared to their associated disks. Fig. 4 reveals that especially at high mass bars are extremely common and likely have a big impact on the average photometric properties of the galaxy population. Therefore bars are strong contributors of the trends in color shown in the template color profiles of $\mathrm{C} 16$ especially for high mass objects. Moreover a further clue comes from the distribution of the ratio between $a_{\mathrm{bar}}$ and $a_{\text {gal }}$ (Fig. 7). This indicates that the average optical extension of the bar is $\sim 0.3 a_{\mathrm{gal}}$, which is consistent with the extension of the intermediate/internal zone identified in the average color radial profiles of $\mathrm{C} 16$ that is on average redder than the outer disk zone.

A possible explanation for such a correspondence between the presence of the bar and the color of the galaxy is that proposed by Gavazzi et al. (2015) who find that the sSFR of mainsequence local galaxies have a downturn at high mass indicating that massive disks have suppressed SSFR with respect to their lower mass counterparts. These authors suggest that the torque exerted onto the gaseous component by the bar funnels the gas inside the corotation radius to the very center of the galaxy where it is rapidly consumed by a burst of star formation. The region within the bar extent is therefore gas depleted and 

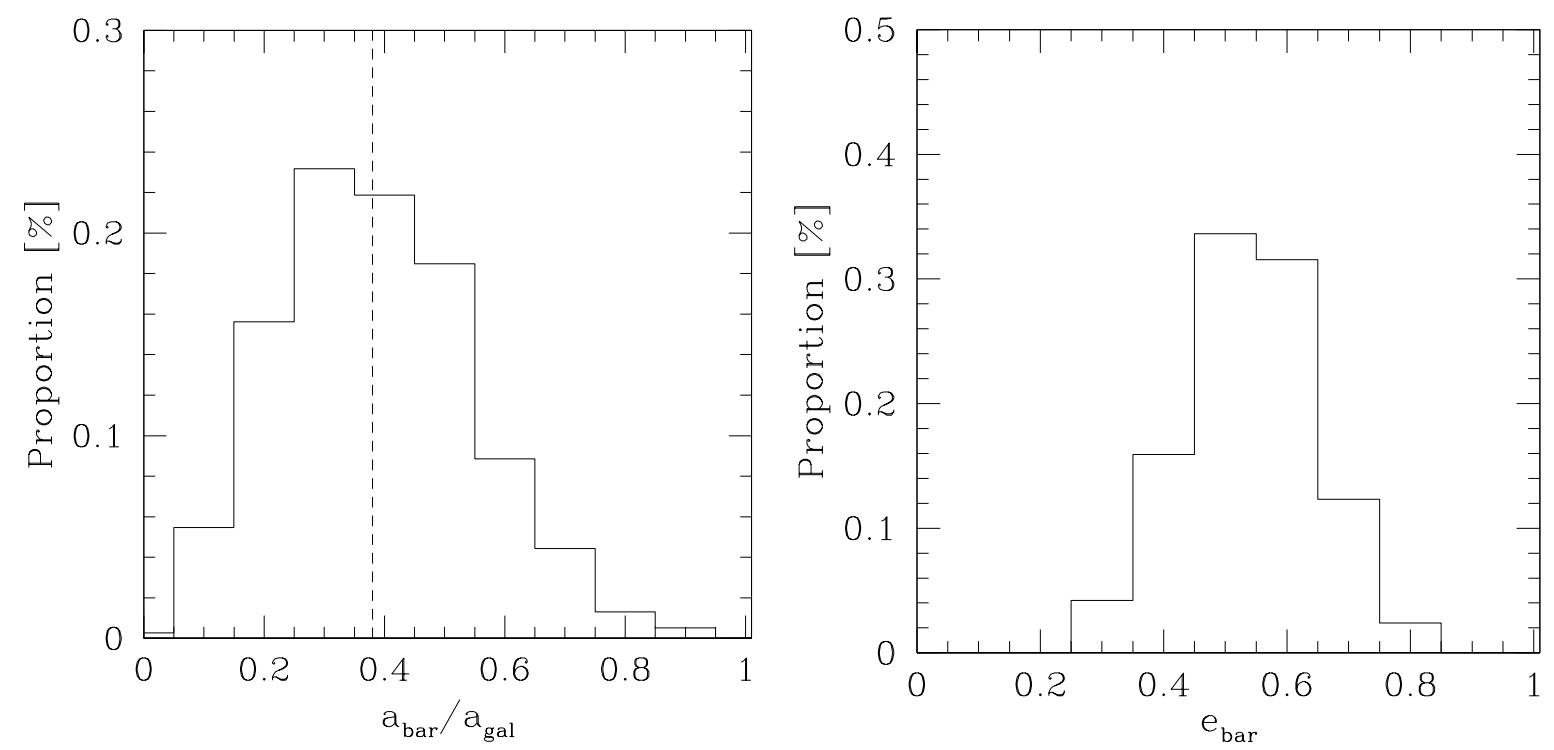

Fig. 7. Left: distribution of the ratio between the deprojected semi-major axis of the bar and the semi-major axis of the galaxy. The dashed line indicates the average value of the distribution equal to 0.38 . Right: the distribution of the ellipticity of the bars extracted in this work.

grows redder with time (see also Hakobyan et al. 2016) and this phenomenon can occur earlier in more massive disks, which are dynamically colder. On the contrary, the gas outside the corotation radius is held in place and keeps feeding the star formation maintaining the disk blue. Nevertheless the bar fraction versus mass relation, along with the well-known color-mass relation (Consolandi et al. 2016), implies that there is a higher fraction of bars among more massive galaxies with redder total colors; however, we stress that these are still star-forming spiral galaxies. This is consistent with previous works such as Masters et al. (2011), Alonso et al. (2013, 2014), who consistently find an increasing bar fraction in redder galaxies.

I found a difference between the bar fraction evaluated among all LTGs (ty $>1$ ) and the bar fraction that embraces lenticulars. As a matter of fact when lenticulars are taken into account the bar fraction decreases at all masses. At this point, a note of caution is required: the morphology selection relies only on visual morphological classification (Binggeli et al. 1985), which cannot disentangle the small population ( $13 \%$ of ETGs, Cappellari et al. 2011; Emsellem et al. 2011) of slow rotators (pure ellipticals) from the much wider population of fast rotators (disks that should have been taken into account when calculating the bar fractions). Therefore this estimate of the bar fraction of the joint population of LTGs and S0/dS0s could be biased by the morphological classification. Nevertheless, the proportion that stands between fast and slow rotators (respectively $\sim 87 \%$ and $\sim 13 \%$ of ETGs, Emsellem et al. 2011) implies that the bar fraction could be even lower, as many fast rotators have been likely classified as ellipticals and therefore excluded from the bar fraction determination. The lower bar fraction can possibly arise from two different scenarios: first, S0s are older systems with respect to other disk galaxies and have already undergone buckling instability that weakened the bar and, second, given the well-known density-morphology relation (Dressler 1980), S0s populate dense environments which prevents them from growing bars because of tidal interactions or fast encounters. Nevertheless, other results suggest that intermediate/high density environments, such as groups, can indeed enhance the possibility of growing a galactic bar (Skibba et al. 2012).

As a final note, I would like to highlight that in the highest mass bin the bar fraction is lower. This feature has a low statistical significance but it can be seen also in other works, such as Nair \& Abraham (2010b), Méndez-Abreu et al. (2012), which show the bar fraction as function of mass. Although this feature has a low significance, such a decrease is possibly consistent with other two possible scenarios. First, more massive disks develop a bar at earlier times with respect to their lower mass counterparts and therefore undergo buckling instability earlier and dismantle the bar at earlier epochs. Second, more massive disks have a different merger history with respect to the low mass population and this may induce a different bar fraction. In the future it would be possible to investigate these hypotheses with the advent of new cosmological simulations at sufficient resolution.

In summary, in this paper I have developed an IDL-based bar finder that performs isophotal fitting on SDSS images and on the basis of the extracted radial ellipticity an PA profiles recognizes barred galaxies, thereby avoiding visual inspection of either the images or the profiles. This procedure makes use of the tasks described in $\mathrm{C} 16$ and has been tested over the same sample in order to evaluate the bar fraction in the Local and Coma superclusters and quantify the influence of barred galaxies on the average properties of color profiles of LTGs shown in C16.

The main points are as follows. (i) The procedure has extracted a fairly pure sample of barred galaxies among faceon LTGs and has led to the calculation of a bar fraction of $\sim 36 \%$, which is consistent with other literature results (Jogee et al. 2004; Marinova \& Jogee 2007; Nair \& Abraham 2010b). (ii) The bar fraction shows a strong mass dependency also obtained by previous works in the local volume and at higher redshifts (Marinova \& Jogee 2007; Nair \& Abraham 2010b; Méndez-Abreu et al. 2012; Oh et al. 2012; Skibba et al. 2012). (iii) The bars that we extracted typically occupy the central $\sim 30-40 \%$ of the host galaxy and is typically strong ( $\sim 90 \%$ of times), which is consistent with the proportions observed by Marinova \& Jogee (2007). (iv) I constructed color average profiles of barred galaxies in different bins of mass and compared these profiles to the template profiles of C16.

Points (ii) and (iii) imply that bars likely have a strong impact on the average color profiles created by $\mathrm{C} 16$, who observed in the template profiles of LTGs the growth of a red and dead component in an intermediate zone inside 0.3 Petrosian radii, whose importance increases with mass. From point (iv) I was 
able to assess that bars are redder structures with respect to their disks and can indeed reproduce the upturn toward red of the templates profiles of $\mathrm{C} 16$. Moreover this further links the presence of a bar to a decrease of the SFR in a disk galaxies as proposed by Cheung et al. (2013), Gavazzi et al. (2015).

Acknowledgements. I thank the anonymous referee for constructive criticism that helped improve the manuscript. I acknowledge Dr. Méndez-Abreu who kindly shared his classification for the comparison to this work and Dr. Massimo Dotti and Prof. Giuseppe Gavazzi for useful discussions. This research has made use of the GOLDmine database (Gavazzi et al. 2003, 2014) and of the NASA/IPAC Extragalactic Database (NED) which is operated by the Jet Propulsion Laboratory, California Institute of Technology, under contract with the National Aeronautics and Space Administration. Funding for the Sloan Digital Sky Survey (SDSS) and SDSS-II has been provided by the Alfred P. Sloan Foundation, the Participating Institutions, the National Science Foundation, the US Department of Energy, the National Aeronautics and Space Administration, the Japanese Monbukagakusho, the Max Planck Society, and the Higher Education Funding Council for England. The SDSS Web site is http://www . sdss.org/. The SDSS is managed by the Astrophysical Research Consortium (ARC) for the Participating Institutions. The Participating Institutions are the American $\mathrm{Mu}-$ seum of Natural History, Astrophysical Institute Potsdam, University of Basel, University of Cambridge, Case Western Reserve University, The University of Chicago, Drexel University, Fermilab, the Institute for Advanced Study, the Japan Participation Group, The Johns Hopkins University, the Joint Institute for Nuclear Astrophysics, the Kavli Institute for Particle Astrophysics and Cosmology, the Korean Scientist Group, the Chinese Academy of Sciences (LAMOST), Los Alamos National Laboratory, the Max-Planck-Institute for Astronomy (MPIA), the Max-Planck-Institute for Astrophysics (MPA), New Mexico State University, Ohio State University, University of Pittsburgh, University of Portsmouth, Princeton University, the United States Naval Observatory, and the University of Washington.

\section{References}

Abazajian, K. N., Adelman-McCarthy, J. K., Agüeros, M. A., et al. 2009, ApJS, 182,543

Aguerri, J. A. L., Méndez-Abreu, J., \& Corsini, E. M. 2009, A\&A, 495, 491

Alonso, M. S., Coldwell, G., \& Lambas, D. G. 2013, A\&A, 549, A141

Alonso, S., Coldwell, G., \& Lambas, D. G. 2014, A\&A, 572, A86

Athanassoula, E. 1992, MNRAS, 259, 345

Athanassoula, E. 2002, in Disks of Galaxies: Kinematics, Dynamics and Perturbations, ASP Conf. Proc., 275, 141

Barazza, F. D., Jogee, S., \& Marinova, I. 2008, ApJ, 675, 1194

Bertin, E., \& Arnouts, S. 1996, A\&AS, 117, 393

Berentzen, I., Heller, C. H., Shlosman, I., \& Fricke, K. 1998, MNRAS, 300, 49

Berriman, G. B., Good, J. C., Laity, A. C., et al. 2004, in Astronomical Data Analysis Software and Systems (ADASS) XIII, ASP Conf. Proc., 314, 593

Binggeli, B., Sandage, A., \& Tammann, G. A. 1985, AJ, 90, 1681

Blanton, M. R., Schlegel, D. J., Strauss, M. A., et al. 2005, AJ, 129, 2562

Cappellari, M., Emsellem, E., Krajnović, D., et al. 2011, MNRAS, 413, 813

Cheung, E., Athanassoula, E., Masters, K. L., et al. 2013, ApJ, 779, 162

Cole, D. R., Debattista, V. P., Erwin, P., Earp, S. W. F., \& Roskar, R. 2014, MNRAS, 445, 3352

Consolandi, G., Gavazzi, G., Fumagalli, M., Dotti, M., \& Fossati, M. 2016, A\&A, 591, A38

Daddi, E., Elbaz, D., Walter, F., et al. 2010, ApJ, 714, L118

de Vaucouleurs, G., de Vaucouleurs, A., Corwin, H. G., Jr., et al. 1991, Third Reference Catalogue of Bright Galaxies, Volume I: Explanations and references, Volume II: Data for galaxies between $0^{\mathrm{h}}$ and $12^{\mathrm{h}}$, Volume III: Data for galaxies between $12^{\mathrm{h}}$ and $24^{\mathrm{h}}$ (New York: Springer)

Dressler, A. 1980, ApJ, 236, 351

Emsellem, E., Cappellari, M., Krajnović, D., et al. 2011, MNRAS, 414, 888

Emsellem, E., Renaud, F., Bournaud, F., et al. 2015, MNRAS, 446, 2468

Eskridge, P. B., Frogel, J. A., Pogge, R. W., et al. 2000, AJ, 119, 536
Fanali, R., Dotti, M., Fiacconi, D., \& Haardt, F. 2015, MNRAS, 454, 3641 Gavazzi, G., Boselli, A., Donati, A., Franzetti, P., \& Scodeggio, M. 2003, A\&A, 400,451

Gavazzi, G., Fumagalli, M., Cucciati, O., \& Boselli, A. 2010, A\&A, 517, A73

Gavazzi, G., Fumagalli, M., Galardo, V., et al. 2012, A\&A, 545, A16

Gavazzi, G., Savorgnan, G., Fossati, M., et al. 2013, A\&A, 553, A90

Gavazzi, G., Franzetti, P., \& Boselli, A. 2014, ArXiv e-prints

[arXiv: 1401.8123]

Gavazzi, G., Consolandi, G., Dotti, M., et al. 2015, A\&A, 580, A116

Genzel, R., Tacconi, L. J., Gracia-Carpio, J., et al. 2010, MNRAS, 407, 2091

Haynes, M. P., Giovanelli, R., Martin, A. M., et al. 2011, AJ, 142, 170

Hakobyan, A. A., Karapetyan, A. G., Barkhudaryan, L. V., et al. 2016, MNRAS, 456, 2848

Hubble, E. P. 1936, Realm of the Nebulae (New Haven: Yale University Press)

Jogee, S., Barazza, F. D., Rix, H.-W., et al. 2004, ApJ, 615, L105

Jogee, S., Scoville, N., \& Kenney, J. D. P. 2005, ApJ, 630, 837

Kim, W.-T., Seo, W.-Y., \& Kim, Y. 2012, ApJ, 758, 14

Knapen, J. H. 1999, in The Evolution of Galaxies on Cosmological Timescales, ASP Conf. Proc., 187, 72

Kormendy, J. 2013, in Secular Evolution of Galaxies (Cambridge, UK: Cambridge University Press), 1

Kormendy, J., \& Kennicutt, R. C., Jr. 2004, ARA\&A, 42, 603

Krumholz, M. R., \& McKee, C. F. 2005, ApJ, 630, 250

Krumholz, M. R., McKee, C. F., \& Tumlinson, J. 2009, ApJ, 699, 850

Lansbury, G. B., Lucey, J. R., \& Smith, R. J. 2014, MNRAS, 439, 1749

Laurikainen, E., Salo, H., Buta, R., Knapen, J. H., \& Comerón, S. 2010, MNRAS, 405, 1089

Lintott, C. J., Schawinski, K., Slosar, A., et al. 2008, MNRAS, 389, 1179

MacArthur, L. A., Courteau, S., Bell, E., \& Holtzman, J. A. 2004, ApJS, 152, 175

Marinova, I., \& Jogee, S. 2007, ApJ, 659, 1176

Marinova, I., Jogee, S., Weinzirl, T., et al. 2012, ApJ, 746, 136

Markwardt, C. B. 2009, in Astronomical Data Analysis Software and Systems XVIII, ASP Conf. Proc., 411, 251

Masters, K. L., Nichol, R. C., Hoyle, B., et al. 2011, MNRAS, 411, 2026

Masters, K. L., Nichol, R. C., Haynes, M. P., et al. 2012, MNRAS, 424, 2180

McDonald, M., Courteau, S., Tully, R. B., \& Roediger, J. 2011, MNRAS, 414, 2055

Melvin, T., Masters, K., Lintott, C., et al. 2014, MNRAS, 438, 2882

Méndez-Abreu, J., Sánchez-Janssen, R., \& Aguerri, J. A. L. 2010, ApJ, 711, L61

Méndez-Abreu, J., Sánchez-Janssen, R., Aguerri, J. A. L., Corsini, E. M., \& Zarattini, S. 2012, ApJ, 761, L6

Menéndez-Delmestre, K., Sheth, K., Schinnerer, E., Jarrett, T. H., \& Scoville, N. Z. 2007, ApJ, 657, 790

Nair, P. B., \& Abraham, R. G. 2010a, ApJS, 186, 427

Nair, P. B., \& Abraham, R. G. 2010b, ApJ, 714, L260

Oh, S., Oh, K., \& Yi, S. K. 2012, ApJS, 198, 4

Regan, M. W., \& Teuben, P. J. 2004, ApJ, 600, 595

Sánchez-Janssen, R., Méndez-Abreu, J., \& Aguerri, J. A. L. 2010, MNRAS, 406, L65

Sanders, R. H., \& Huntley, J. M. 1976, ApJ, 209, 53

Sakamoto, K., Okumura, S. K., Ishizuki, S., \& Scoville, N. Z. 1999, ApJ, 525, 691

Sheth, K., Vogel, S. N., Regan, M. W., Thornley, M. D., \& Teuben, P. J. 2005 , ApJ, 632, 217

Sheth, K., Elmegreen, D. M., Elmegreen, B. G., et al. 2008, ApJ, 675, 1141

Sellwood, J. A. 2014, Rev. Mod. Phys., 86, 1

Shlosman, I., Frank, J., \& Begelman, M. C. 1989, Nature, 338, 45

Skibba, R. A., Masters, K. L., Nichol, R. C., et al. 2012, MNRAS, 423, 1485

Thompson, L. A. 1981, ApJ, 244, L43

Willett, K. W., Lintott, C. J., Bamford, S. P., et al. 2013, MNRAS, 435, 2835

Wozniak, H., Friedli, D., Martinet, L., Martin, P., \& Bratschi, P. 1995, A\&AS, 111,115

York, D. G., Adelman, J., Anderson, J. E., Jr., et al. 2000, AJ, 120, 1579

Zibetti, S., Charlot, S., \& Rix, H.-W. 2009, MNRAS, 400, 1181

Zwicky, F., Herzog, E., \& Wild, P. 1968, Catalogue of galaxies and of clusters of galaxies (Pasadena: California Institute of Technology) 